\title{
Energy landscapes for mobile ions in ion conducting solids
}

\author{
S ADAMS \\ Department of Materials Science and Engineering, National University of Singapore, 117576, Singapore
}

\begin{abstract}
Structure property function relationships provide valuable guidelines in the systematic development of advanced functional materials with tailored properties. It is demonstrated that an augmented bond valence approach can be effectively used to establish such relationships for solid electrolytes. A bond valence analysis of local structure models for disordered systems or interfaces based on reverse Monte Carlo (RMC) fits or molecular dynamics (MD) simulations yields quantitative predictions of the ion transport characteristics. As demonstrated here for a range of metaphosphate and diborate glasses, the complete description of the energy landscape for mobile ions also provides an effective tool for achieving a more detailed understanding of ion transport in glasses. The investigation of time evolutions can be included, if the bond valence analysis is based on MD trajectories. In principle, this allows quantifying the time and temperature dependence of pathway characteristics, provided that a suitable empirical force-field is available. For the example of $\mathrm{LiPO}_{3}$, the remaining differences between simulated and experimental structures are investigated and a compensation method is discussed.
\end{abstract}

Keywords. Solid electrolytes; bond valence analysis; ion transport in glasses.

\section{Introduction}

In order to understand ion conduction in glassy electrolytes, it is essential to explore the relationship between conductivity and structural peculiarities. Structural information can be obtained experimentally by a wide range of techniques: mainly neutron and X-ray diffraction for general information as well as NMR, EXAFS and vibrational spectroscopy for local information on specific parts of the structure. Presently, the only viable method to generate structure models from experimental data is by reverse Monte Carlo (RMC) fitting. RMC produces static models that quantitatively agree with diffraction, EXAFS and NMR data, as well as additional bonding constraints based on further experimental or chemical knowledge (Keen and McGreevy 1990; McGreevy 1995, 2001; Swenson et al 1997). In this way experimental data can be translated into a consistent model of distribution and local environment of the potentially mobile ions, an essential step towards an understanding of the conduction process. Still it has to be emphasized that RMC models are no unique structure solutions and their interpretation is thus limited to a statistical extraction of characteristic features.

In previous work we discussed how the bond valence method can be utilized to perform such a statistical analysis of ion transport pathways within the RMC models yielding predictions of ionic conductivity (see e.g. Adams and Swenson 2000, 2002, 2004, 2005; Swenson and Adams 2003). In this work, investigation of ion transport mechanisms is extended by discussing the number, distribution

(mseasn@nus.edu.sg) and connectivity of 'equilibrium sites' (relaxed sites) for the mobile type of ions in various metaphosphate and diborate glasses.

Alternatively the relation between structure and conductivity can be investigated using molecular dynamics (MD) simulations, which should, in principle, permit to derive all the required structural and dynamical information (within the limitations imposed by the system size and the simulated period). Effectively, the MD approach has been shown to be a useful tool in obtaining insight into the conduction mechanism and its correlation to the atomic structure (see e.g. Balasubramanian and Rao 1995; Habasaki and Hiwatari 1999; Jund et al 2001; Yuan and Cormack 2003). Besides the computational effort required to reach simulations time scales exceeding the nanoseconds regime for the inevitably complex structure models, the most serious limitation is the problem to obtain realistic interatomic potentials between the particles in chemically complicated glasses that quantitatively reproduce experimentally known structural or dynamical properties of the glasses. Here we discuss a comparison of RMC models for glassy $\mathrm{LiPO}_{3}$ with a MD simulation of the same material to elucidate the possibility of using MD simulations or a combination of RMC and MD as the basis for a bond valence analysis of the time evolution of ion transport pathways.

\section{Techniques}

Details of the slightly modified reverse Monte Carlo method used to build static structure models have recently been 
described elsewhere (Adams and Swenson 2005). For comparison, a dynamic structure model of $\mathrm{LiPO}_{3}$ of the same size (4000 atoms, NVT, $T=300 \mathrm{~K}$ ) has been generated by MD simulations using an established 3-body force field (Alam et al 2000) starting from the RMC configuration and keeping the experimentally known density fixed. The analyses of ion transport pathways in the RMC model as well as in a set of 100 snapshots from the MD trajectory have been performed using a bond valence approach.

Empirical relationships between bond length and bond valence (BV) are widely used to identify plausible equilibrium sites for an atom in a crystal structure as sites where the BV sum of the atom matches its oxidation state (cf. e.g. Brown 2002). A modification of the BV approach that improves the assessment of non-equilibrium site energies by a systematic adjustment of BV parameters to the bond softness (Adams 2001, 2003, 2006; Adams and Swenson 2004) extends the application range of this simple tool to structure-property relationship studies in solid electrolytes. For that purpose, 'accessible' sites for a mobile ion, $A$, in a local structure model are identified using empirical relationships between the bond length, $R$ and a so-called bond valence, $s_{A-X}$,

$$
s_{A-X}=\exp \left[\left(R_{0}-R\right) / b\right],
$$

as sites where the mismatch of the bond valence sum, $V(A)$, is

$$
|\Delta V(A)|=\left|\sum_{X} S_{A-X}-V_{\text {id }}(A)\right|+\sum_{X} p_{A-X},
$$

over the $s_{A-X}$ from all adjacent counterions, $X$ approaches the ideal valence, $V_{\text {id }}(A)$ (which equals its oxidation state). To enhance the chemical plausibility of $\mathrm{BV}$ mismatch 'energy landscapes', $|\Delta V(A)|$ contains a penalty function, $p_{A-X}$, that discriminates against sites where a matching $V(A)$ is achieved by unfavourable strongly asymmetric coordinations (for details see Adams 2006) and is complemented by hard minimum distance and soft coordination number constraints. In contrast to most conventional BV parameter sets our soft BV parameters are based on the assumption that not only the counterions of the first coordination shell but all counterions up to a cut-off radius of 4-8 $\AA$ (depending on sizes and polarizabilities of the ions involved) contribute to $V(A)$. This is indispensable for modelling ion transport pathways as it avoids artefacts in the BV sum variation, when an ion moves across the border of its coordination shell.

$\mathrm{BV}$ ion transport pathway models are based on the supposition that paths between accessible sites, along which $|\Delta V(A)|$ remains sufficiently low represent probable ion transport paths. Isosurfaces of fixed maximum BV sum mismatch $|\Delta V(A)|$ for a certain ion type $A$ will thus enclose regions that an $A$ ion in the pathway may reach with a certain activation energy, $E_{\mathrm{a}}$. Low $\mathrm{BV}$ mismatch regions that include both occupied and vacant sites, there- fore, enable local jumps of $A$. A long-range transport requires pathways that extend through the whole structure. For periodic models this is equivalent to spanning the model box.

Determining the 'pathway volume' for a given maximum value of $|\Delta V|$ yields a simple and reliable way of characterizing ion transport pathways in local structure models of disordered systems (Adams and Swenson 2002, 2004). To evaluate this pathway volume, $V(A)$, has to be calculated for a hypothetical $A^{+}$ion at each point in the structure. In practice, the structure model (containing typically 4000 atoms) is divided into a primitive grid containing $\sim 4$ million cubic volume elements with a size of $(0 \cdot 2-0 \cdot 3 \AA)^{3}$. A volume element is considered to belong to the conduction pathway if $\left|V(A)-V(A)_{\text {ideal }}\right|$ is below a fixed threshold value or if the sign of $\left(V-V_{\text {ideal }}\right)$ alters within the volume element (The second condition cushions effects of the limited grid resolution). Accessible volume elements that share common faces or edges belong to the same 'pathway cluster'. It is assumed that these pathway clusters contribute to d.c. conductivity only if they percolate through the structure model. The volume fraction, $F$, of the percolating pathways turns out to be a suitable measure of $E_{\mathrm{a}}$ and thereby (in the case of glasses) also of the room temperature conductivity. The remaining restricted pathway clusters are considered to contribute only to a.c. conductivity. As mentioned above, the threshold value for $|\Delta V|$ has to be chosen somewhat arbitrarily. When comparing pathways for different types of mobile ions, we find that the most suitable value will, as discussed further below, depend on the mass, $m_{A}$, of the mobile ion.

\section{Results and discussion}

\subsection{Enumeration of sites for mobile ions in metaphosphate glasses}

In contrast to ion transport in crystalline solid electrolytes that is well understood in terms of interstitial, interstitialcy or vacancy mechanisms, there is a continuing debate whether comparable conduction mechanisms prevail in glasses. As a first step towards a deeper insight, it appears indispensable to count (and classify) all sites that a mobile ion can visit in a given glass structure. Such attempts have been undertaken previously on the basis of molecular dynamics trajectories for a number of glass systems using different routines to identify suitable sites (see for e.g. Lammert et al 2003). Identifying those sites that have actually been occupied by the mobile ions for a certain while, however, results in the fundamental uncertainty that the resulting number will depend on the simulated period. This becomes a serious drawback for systems with low cation mobilities such as mixed alkali glasses. The detection of 'equilibrium sites' for RMC models of various metaphosphate glasses, $\mathrm{APO}_{3}$ (where $\mathrm{A}^{+}=\mathrm{Li}^{+}, \mathrm{Na}^{+}, \mathrm{Rb}^{+}$, 
$\mathrm{Ag}^{+}$), $\mathrm{AgI}$-doped $\mathrm{AgPO}_{3}$ and halide doped alkali diborate glasses using the bond valence method may, however, be (approximately) identified with an analysis of the complete energy landscape for the modelled local structure snapshot at a certain instant. As glasses are always metastable states, the term 'equilibrium sites' only refers to the fact that these sites are energy minima for the $\mathrm{A}^{+}$for the frozen anion arrangement. The method, therefore, yields a complete inventory of all available sites for $\mathrm{A}^{+}$at a given instant irrespective of their occupancy, but, of course, lacks the information on time evolution.

For each of the systems the starting point is the identification of 'accessible' sites within the RMC structure model. To account for the above-mentioned mass dependence of the conversion between activation energy and bond valence mismatch, we employed a valence mismatch threshold of 0.026 v.u./amu ${ }^{1 / 2} \cdot m_{M}{ }^{1 / 2}$ (v.u. = valence units; $\mathrm{amu}=$ atomic mass unit), which leads to valence mismatch thresholds ranging from $0.068 \mathrm{v}$.u. (for $\mathrm{Li}$ ) to $0 \cdot 27$ v.u. (for $\mathrm{Ag}$, cf. table 1). Due to the limited grid resolution using much smaller valence mismatch thresholds would bear the danger of missing some of the local bond valence sum minima.

For the investigated glasses the condition of a sufficiently low bond valence mismatch will not produce individual sites but a continuous network of sites with only marginal differences in their bond valence mismatch. The bond valence approach suggests that if a cation can reach the appropriate bond valence sum in various ways it will prefer the coordination that leads to the most uniform distribution of individual bond valences (equal valence rule, Brown 2002). Together with the knowledge that the coordination number $(\mathrm{CN})$ for equilibrium sites of a certain cation in oxide salts will vary only over a limited range this can be used to resolve the pathways into equilibrium sites and jump paths between these sites.
In accordance to a suggestion by Brown (2002), anions, $\mathrm{X}$, are classified as coordinating a monovalent cation, $A$, if the $A-X$ distance corresponds to an individual bond valence $s_{A-X} \geq 0.04$ v.u. A survey of cation coordinations in sets of well-determined, fully ordered inorganic crystal structures extracted from the Inorganic Crystal Structure database using this $\mathrm{CN}$ definition demonstrates that the ranges of preferred $\mathrm{CN}$ for $\mathrm{Li}^{+}, \mathrm{Na}^{+}, \mathrm{Rb}^{+}$and $\mathrm{Ag}^{+}$in oxides and oxyhalides are 4-6, 4-6, 4-8 and 2-6, respectively. Only sites that lead to a $\mathrm{CN}$ within this range qualify as equilibrium sites. As none of the investigated cations tends to form multiple bonds, sites leading to individual bond valences significantly $>1 / 4$ for the alkali glasses or $>1 / 2$ for the Ag glasses are also eliminated. For alkali glasses these criteria locate clusters of equilibrium sites that are sufficiently small (about 10 grid sites or corresponding to an equivalent radius of the site of $0.4 \AA$, cf. table 1 ) and thus can only be occupied by a single cation. (This size should not be mistaken with the ion radius, which is already accounted for implicitly when calculating the BV sum. It rather describes the variability of the position for the centre of the ion and thus resembles an atomic displacement parameter.) Due to the tendency of silver ions to occur in low $\mathrm{CN}$ configurations (which imposes the less strict coordination number constraint $2 \leq \mathrm{CN} \leq 6$ ), the same formalism leads to considerably larger 'equilibrium site clusters' in silver ion conducting glasses that may be occupied by several $\mathrm{Ag}^{+}$at the same time. Thereby the values found for the $\mathrm{Ag}^{+}$conducting glasses are not directly comparable. On the other hand this also suggests a higher degree of correlation between the elementary hop processes in $\mathrm{Ag}^{+}$ion conducting glasses. The formalism described so far does not take into account, whether all 'equilibrium site' clusters can be occupied simultaneously. Effectively the criteria (applied to a grid with limited resolution) will produce a considerable number of closely

Table 1. Enumeration of 'equilibrium' site clusters (relaxed sites) for the mobile ions in various metaphosphate or diborate glasses.

\begin{tabular}{|c|c|c|c|c|c|c|}
\hline & \multicolumn{2}{|c|}{ Criteria } & \multicolumn{4}{|c|}{ Equilibrium site clusters } \\
\hline & $|\Delta V|$ (v.u.) & $\mathrm{CN}$ (range) & Sites/ions & $\begin{array}{l}\text { Av. site radius } \\
(\AA)\end{array}$ & $\begin{array}{l}\text { Simultaneously } \\
\text { accessible }\end{array}$ & $\begin{array}{c}\text { Simultaneously } \\
\text { accessible eq. sites/ion }\end{array}$ \\
\hline $\mathrm{LiPO}_{3}$ & $0 \cdot 068$ & $4-6$ & $2954 / 800$ & $0 \cdot 32$ & 1339 & $1 \cdot 67$ \\
\hline $\mathrm{NaPO}_{3}$ & $0 \cdot 125$ & $4-6$ & $2084 / 800$ & $0 \cdot 41$ & 872 & $1 \cdot 09$ \\
\hline $\mathrm{RbPO}_{3}$ & $0 \cdot 240$ & $4-8$ & $1693 / 800$ & $0 \cdot 42$ & 556 & $0 \cdot 70$ \\
\hline $\mathrm{Li}_{0.5} \mathrm{Na}_{0.5} \mathrm{PO}_{3}\left(\mathrm{Li}^{+}\right)$ & $0 \cdot 068$ & $4-6$ & $1396 / 400$ & $0 \cdot 28$ & 631 & $1 \cdot 58$ \\
\hline $\mathrm{Li}_{0.5} \mathrm{Na}_{0.5} \mathrm{PO}_{3}\left(\mathrm{Na}^{+}\right)$ & $0 \cdot 125$ & $4-6$ & $819 / 400$ & $0 \cdot 34$ & 383 & $0 \cdot 96$ \\
\hline $\mathrm{AgPO}_{3}$ & $0 \cdot 270$ & $2-6$ & $(154 / 768)$ & $1 \cdot 75$ & 1018 & $1 \cdot 33$ \\
\hline $10 \mathrm{AgI}-90 \mathrm{AgPO}_{3}$ & $0 \cdot 270$ & $2-6$ & $(214 / 853)$ & $1 \cdot 60$ & 1193 & $1 \cdot 40$ \\
\hline $30 \mathrm{AgI}-70 \mathrm{AgPO}_{3}$ & $0 \cdot 270$ & $2-6$ & $(314 / 1000)$ & $1 \cdot 44$ & 1533 & $1 \cdot 53$ \\
\hline $50 \mathrm{AgI}-50 \mathrm{AgPO}_{3}$ & $0 \cdot 270$ & $2-6$ & $(178 / 1200)$ & $2 \cdot 23$ & 1902 & 1.59 \\
\hline $\mathrm{Li}_{2} \mathrm{O}-2 \mathrm{~B}_{2} \mathrm{O}_{3}$ & 0.068 & $4-6$ & $2201 / 600$ & $0 \cdot 36$ & 1023 & $1 \cdot 71$ \\
\hline $\mathrm{LiCl}-\mathrm{Li}_{2} \mathrm{O}-2 \mathrm{~B}_{2} \mathrm{O}_{3}$ & $0 \cdot 068$ & $4-6$ & $2508 / 720$ & $0 \cdot 38$ & 1286 & 1.79 \\
\hline $\mathrm{Na}_{2} \mathrm{O}-2 \mathrm{~B}_{2} \mathrm{O}_{3}$ & $0 \cdot 125$ & $4-6$ & $1045 / 600$ & $0 \cdot 46$ & 570 & $0 \cdot 95$ \\
\hline
\end{tabular}


neighbouring clusters and thereby overestimate the number of relevant sites. A more relevant quantity is the number, $n_{\text {site }}$, of 'equilibrium sites' that have a suitable minimum distance from each other to be occupied independently. $n_{\text {site }}$ is assessed by running systematically through all sites. Wherever an accessible site within an equilibrium site cluster is identified, all surrounding sites up to a distance of $2 r_{A}$ are eliminated before continuing the loop. Appropriate $A-A$ exclusion distances, $2 r_{A}$, are again derived from sets of reliable reference crystal structures that contain short $A-A$ distances (for details, see Adams and Swenson 2005). The resulting exclusion radii, $r_{\mathrm{Li}}=1.17 \AA, r_{\mathrm{Na}}=r_{\mathrm{Ag}}=1.38 \AA$ for $\mathrm{Na}$ and $\mathrm{Ag}$ and $r_{\mathrm{Rb}}=$ $1.85 \AA$ are slightly smaller than the respective metallic radii and consistent with the minimum distance criteria employed in the RMC modelling of the investigated systems.

Although the choice of threshold value $|\Delta V|$ is guided by the considerations discussed above (e.g. regarding the systematic mass dependence, limitations by grid resolution, etc), it remains to some extent arbitrary. Therefore, it needs to be tested to which extent results depend on a specific choice of $|\Delta V|$. Results for the alkali metaphosphate glasses are summarized in figure 1, where characteristic properties of the pathways and 'equilibrium sites' are shown as a function of the chosen value of $|\Delta V|$. In each graph the (ion mass dependent) value selected for the analysis in table 1 is marked by a broken vertical line. When $\Delta V$ is raised the pathway volume fraction increases nearly linearly (after an initial plateau region, where the pathway volume is mainly determined by the sign change criterion; figure 1i). The number of clusters forming the pathways goes through a maximum for $\Delta V$ values that for all investigated systems are smaller than the chosen threshold value (figure 1ii). The ratio between the number of simultaneously accessible 'equilibrium sites' for $\mathrm{A}^{+}$ and the number of $\mathrm{A}^{+}$in the structure model varies only slowly with the choice of $\Delta V$ (figure 1iii). Thereby, the conclusion that the mechanism for ion transport will depend on the type of mobile ion, is hardly affected by the choice of a particular threshold value for $\Delta V . \mathrm{Li}^{+}$ion transport in $\mathrm{LiPO}_{3}$ occurs via a significant fraction of unoccupied sites, while unoccupied $\mathrm{Rb}^{+}$sites are rare in $\mathrm{RbPO}_{3}$ so that ion transport resembles more a 'vacancy'type mechanism.

To control the relevance of the pathway clusters, figure liv indicates the correlation between pathway sites (that as shown in figure $1 \mathrm{i}$ contain only $\sim 1 \%$ of the total volume of the structure model) and the cation sites of the RMC model. Again grid resolution limits the precision of this analysis. Therefore, three variants of the correlation are plotted: Curve (a) in figure 1iv displays the percentage of modelled $\mathrm{A}^{+}$sites that are located within one of the accessible volume elements; while versions (b) and (c) additionally
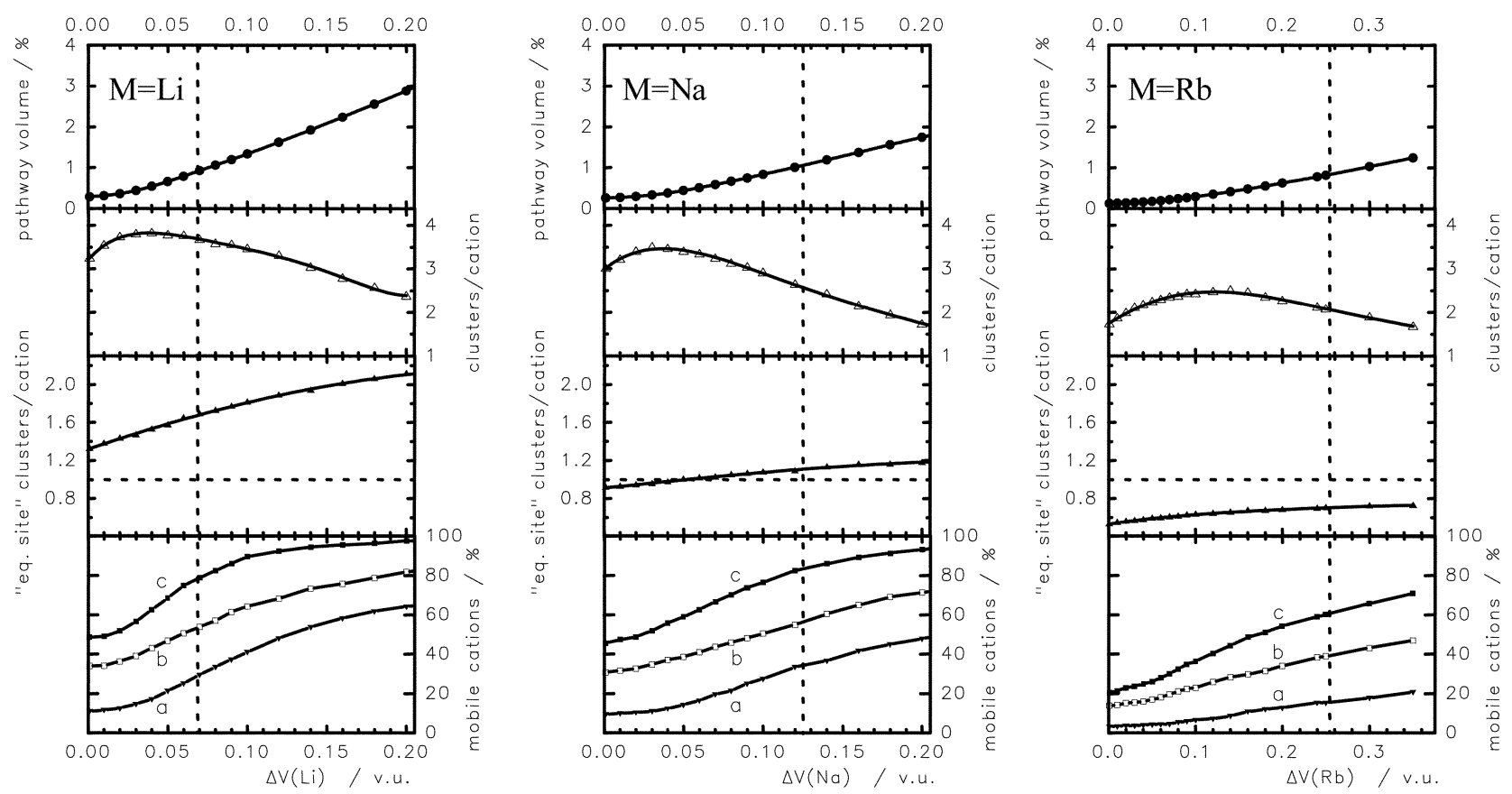

Figure 1. Variation of quantities characterizing the bond valence pathways in reverse Monte Carlo models of $\mathrm{MPO}_{3}$ glasses $(\mathrm{M}=\mathrm{Li}, \mathrm{Na}$ or $\mathrm{Rb}$ from left to right) as a function of the chosen bond valence mismatch threshold: (i) pathway volume fraction; (ii) number of clusters per cation; (iii) number of 'equilibrium site clusters' per cation and (iv) percentage of $\mathrm{M}^{+}$ions that are located (a) within volume elements belonging to the pathway, (b) in volume elements that belong to the pathway or their face sharing neighbours and (c) in volume elements that belong to the pathway or their face or edge-sharing neighbours. The dashed vertical line indicates the mass-dependent threshold value for $|\Delta V|$ chosen in this work. 


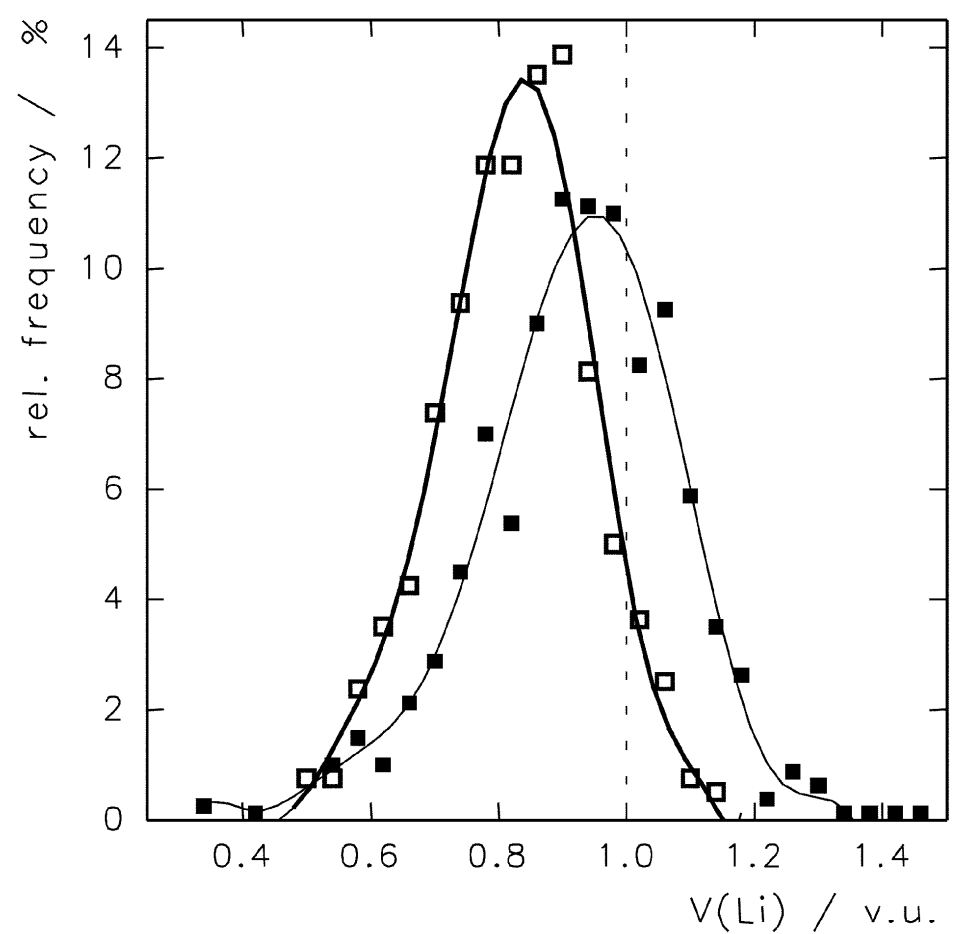

Figure 2. Bond valence sum distribution of $\mathrm{Li}$ atoms in a $\mathrm{RMC}$ model of glassy $\mathrm{LiPO}_{3}$ (filled symbols) as well as in a series of snapshots from a molecular dynamics simulation of $\mathrm{LiPO}_{3}$ at $T=300 \mathrm{~K}$ (open symbols). Solid lines as a guide to the eye.

include the face-sharing or face- and edge-sharing neighbours of accessible sites. Even for curve (c) this means that the $\mathrm{BV}$ pathway is within the region that the $\mathrm{A}^{+}$probes by its thermal vibrations and such a $\mathrm{A}^{+}$should therefore be considered as mobile. For $\mathrm{LiPO}_{3}$ and $\mathrm{NaPO}_{3}$ this includes $\sim 80 \%$ of the alkali ions, while the number is reduced to $\sim 60 \%$ for the $\mathrm{RbPO}_{3}$ glass.

\subsection{Comparison of pathways in RMC and MD- generated structure models of $\mathrm{LiPO}_{3}$}

To elucidate the possibilities of applying our bond valence analysis formalism to structure models from MD simulations, the characteristics of $\mathrm{Li}^{+}$pathways in RMC- and MD-generated structure models of glassy $\mathrm{LiPO}_{3}$ are compared. In the case of the MD model results are averaged over 100 snapshots from a short trajectory (30 ps) after a relaxed state has been reached in a previous simulation run. The analysis of coordination number distributions for the $\mathrm{Li}^{+}$in both models seems to emphasize the similarity of both structure models $(\mathrm{CN}(\mathrm{Li})=4.49 \pm 0.85$ from $\mathrm{MD}$ simulations, $4.57 \pm 1.07$ ), but the broader distribution already hints at the slightly more disordered nature of RMC models. Figure 2, however, reveals potential problems for a straightforward application of BV analysis to the MD model: the average $\mathrm{BV}$ sum $\mathrm{V}(\mathrm{Li})$ for the $\mathrm{Li}$ atoms in the MD model $\mathrm{V}(\mathrm{Li})=0 \cdot 826 \pm 0 \cdot 121 \mathrm{v}$.u. is significantly lower than $\mathrm{V}(\mathrm{Li})=0.927 \pm 0.154$ v.u. for the RMC model. Average BV sums slightly below $V_{\text {id }}=1$ v.u. for monovalent cations are to be expected and generally observed in RMC structure models due to the snapshot type nature of the models (BV parameters are determined from stable, well-ordered reference structures, where the timeaveraged cation positions represent local energy minima). Still the displayed example indicates that the suitability of a MD simulation force field for this kind of analysis has to be verified in every case.

For minor BV sum deviations adapting the reference value, $V_{\text {id }}$, may be a practical workaround. The influence of such adaptations on the characteristic quantities for the Li pathways in both types of structure models is shown as a function of $|\Delta V|=\left|V-V_{\text {id }}\right|$ in figure 3 for three choices of $V_{\text {id }}, 0.825,0.9$ and 1 valence units (v.u.), and two values, 0.925 and $1 \mathrm{v} . \mathrm{u}$. for the RMC model. As to be expected the pathway volume fraction is highest if $V_{\text {id }}$ is chosen in accordance to the maximum of the respective BV sum distribution. $V_{\text {id }}=1 \mathrm{v} . \mathrm{u}$. is not a suitable choice for the MD case, but the similarity in the $|\Delta V|$ dependence for the remaining four curves implies that an approximate conversion between the most suitable $V_{\text {id }}$ for RMC and MD can be found for a given force field. For any of the choices 

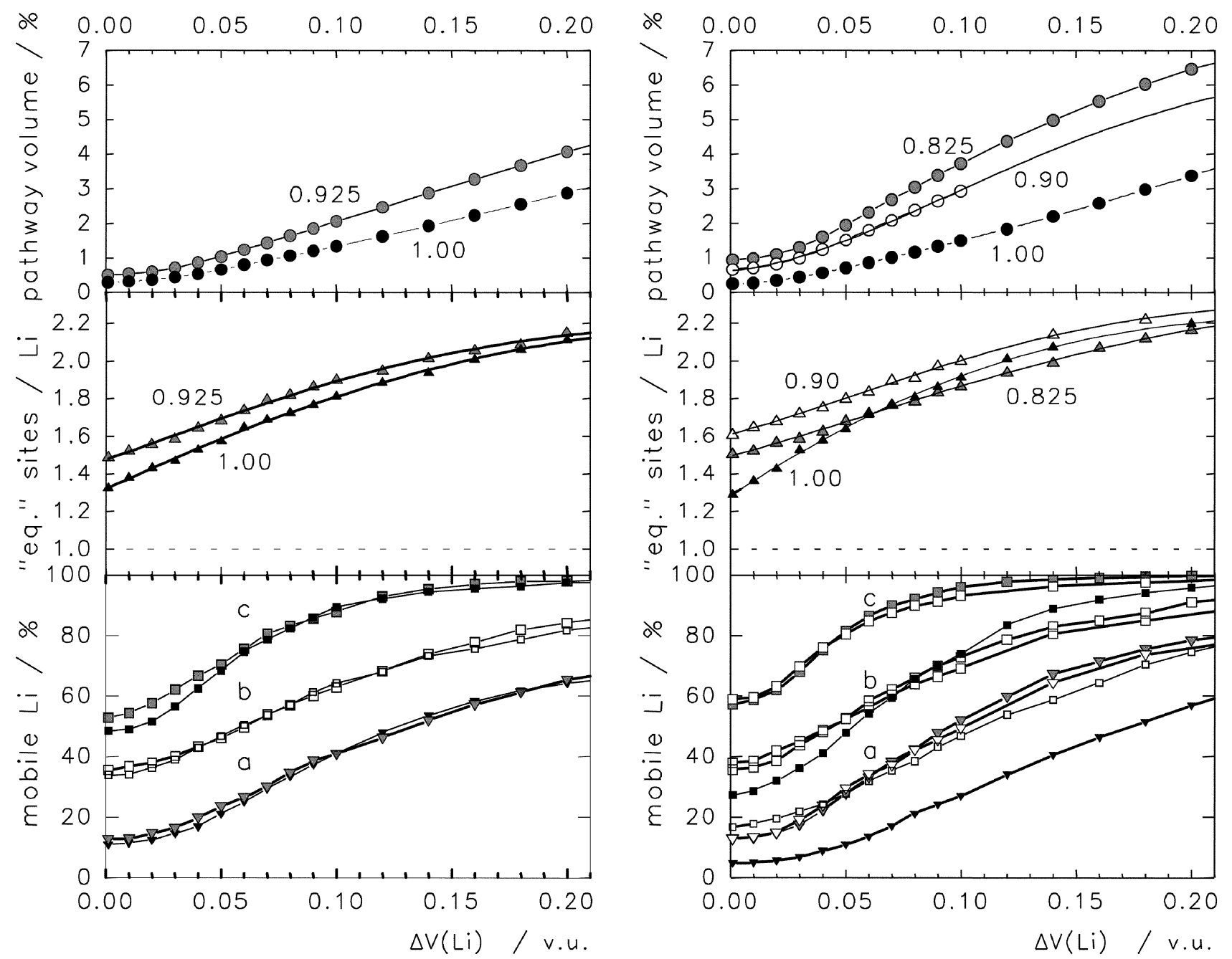

Figure 3. Comparison of bond valence pathway models for $\mathrm{LiPO}_{3}$ based on $\mathrm{RMC}$ models (1.h.s.) or MD simulations (r.h.s.) for different choices of the ideal bond valence sum, $V_{\text {id }}$, with respect to (i) percentage of 'accessible' volume elements; (ii) number of sites with matching BV sum and CN, divided by number of Li in model and (iii) percentage of Li in model, for which (a) the respective volume element itself, (b) the volume element or its face-sharing neighbour and (c) the respective volume element or its face- or edge-sharing neighbour is 'accessible'.

of structure model, $V_{\text {id }}$ and $|\Delta V|$, the number of 'equilibrium sites' for $\mathrm{Li}^{+}$significantly exceeds the number of $\mathrm{Li}^{+}$ions in the structure, so that there are always free target sites for hopping processes. The percentage of mobile Li, which measures the degree of congruency between the $\mathrm{Li}$ positions of the structure model and the BV pathways leads to unsatisfactory results for the MD structure model and $V_{\text {id }}=1 \mathrm{v}$.u. The slight overestimation of $\mathrm{Li}-\mathrm{O}$ bond lengths by the MD force field can be compensated by assuming a slightly smaller $V_{\text {id }}$ in the analysis.

\section{Conclusions}

The application of the bond valence analysis to static structure models from reverse Monte Carlo fits (based on experimental diffraction data) has been demonstrated to provide predictions of ion transport properties. Although an exact localization of the most suitable sites for the mobile ions in local structure models may require a higher resolution than could be applied in the present study, the study still provides semiquantitative results regarding differences in the number of available sites for the mobile ions and thereby in the transport mechanisms for glassy solid electrolytes.

The application to molecular dynamics simulation trajectories adds the possibility to systematically analyse time, temperature or pressure dependencies of energy landscapes for the mobile ions, but characteristics of the respective structure models have to be kept in mind: Cations in MD snapshots have a narrower distribution of $\mathrm{BV}$ sums and coordination numbers than RMC models, 
but (depending on the force field) the average BVS of the mobile ions may differ from the oxidation state. For minor deviations an adaptation of the expected bond valence sum to the specific MD force-field will allow an application of the BV analysis formalism.

\section{References}

Adams S 2001 Acta Crystallogr. B, Struct. Sci. 57278

Adams S 2003 softBV web pages: http://kristall.uni-mki.gwdg. de/softBV/

Adams S 2006 Solid State Ionics (to appear)

Adams S and Swenson J 2000 Phys. Rev. Lett. 84 4144; Phys. Rev. B63 054201

Adams S and Swenson J 2002 Phys. Chem. Chem. Phys. 43179

Adams S and Swenson J 2004 Solid State Ionics 175665

Adams S and Swenson J 2005 J. Phys.: Condens. Matter 17 S87
Alam T M, Liang J J and Cygan R T 2000 Phys. Chem. Chem. Phys. 24427

Balasubramanian S and Rao K J 1995 J. Non-Cryst. Solids 181 157

Brown I D 2002 The chemical bond in inorganic chemistryThe bond valence model (New York: Oxford University Press)

Habasaki J and Hiwatari Y 1999 Phys. Rev. B59 6962

Inorganic Crystal Structure Database 1997 Fachinformationszentrum Karlsruhe, Germany

Jund P, Kob W and Jullien R 2001 Phys. Rev. B64 134303

Keen D A and McGreevy R L 1990 Nature 344423

Lammert H, Kunow M and Heuer A 2003 Phys. Rev. Lett. 90 215901

McGreevy R L 1995 Nucl. Instrum. Meth. in Phys. Res. A354 1

McGreevy R L 2001 J. Phys.: Condens. Matter 13 R877

Swenson J and Adams S 2003 Phys. Rev. Lett. 90155507

Swenson J, Börjesson L, McGreevy R L and Howells W S 1997 Phys. Rev. B55 11236

Yuan X and Cormack A N 2003 J. Non-Cryst. Solids 31931 